\title{
Trabecular bone texture analysis of conventional radiographs in the assessment of knee osteoarthritis: review and viewpoint
}

\author{
Ahmad Almhdie-Imjabbar 1,2, Pawel Podsiadlo3, Richard Ljuhar ${ }^{4}$, Rachid Jennane ${ }^{1,2}$, Khac-Lan Nguyen $^{1,2}$, \\ Hechmi Toumi ${ }^{1,2,5}$, Simo Saarakkala ${ }^{6,7}$ and Eric Lespessailles ${ }^{1,2,5^{*}}$ (i)
}

\begin{abstract}
Background: Trabecular bone texture analysis (TBTA) has been identified as an imaging biomarker that provides information on trabecular bone changes due to knee osteoarthritis (KOA). Consequently, it is important to conduct a comprehensive review that would permit a better understanding of this unfamiliar image analysis technique in the area of KOA research.

We examined how TBTA, conducted on knee radiographs, is associated to (i) KOA incidence and progression, (ii) total knee arthroplasty, and (iii) KOA treatment responses. The primary aims of this study are twofold: to provide (i) a narrative review of the studies conducted on radiographic KOA using TBTA, and (ii) a viewpoint on future research priorities.

Method: Literature searches were performed in the PubMed electronic database. Studies published between June 1991 and March 2020 and related to traditional and fractal image analysis of trabecular bone texture (TBT) on knee radiographs were identified.

Results: The search resulted in 219 papers. After title and abstract scanning, 39 studies were found eligible and then classified in accordance to six criteria: cross-sectional evaluation of osteoarthritis and non-osteoarthritis knees, understanding of bone microarchitecture, prediction of KOA progression, KOA incidence, and total knee arthroplasty and association with treatment response. Numerous studies have reported the relevance of TBTA as a potential bioimaging marker in the prediction of KOA incidence and progression. However, only a few studies have focused on the association of TBTA with both OA treatment responses and the prediction of knee joint replacement.
\end{abstract}

Conclusion: Clear evidence of biological plausibility for TBTA in KOA is already established. The review confirms the consistent association between TBT and important KOA endpoints such as KOA radiographic incidence and progression. TBTA could provide markers for enrichment of clinical trials enhancing the screening of KOA progressors. Major advances were made towards a fully automated assessment of KOA.

Keywords: Trabecular bone texture, Knee osteoarthritis, Conventional radiography

*Correspondence: eric.lespessailles@chr-orleans.fr

1 EA 4708- I3MTO Laboratory, University of Orleans, Orleans, France

Full list of author information is available at the end of the article

\section{Introduction}

Osteoarthritis (OA) is the most common type of arthritis and is among the leading cause of impaired mobility and chronic pain, affecting almost half of the population aged 65 years or older worldwide [1].OA is considered as the most prevalent disorder of articulating joints in humans [2]. Knee, hip, and hand OA are the most common forms original author(s) and the source, provide a link to the Creative Commons licence, and indicate if changes were made. The images or other third party material in this article are included in the article's Creative Commons licence, unless indicated otherwise in a credit line to the material. If material is not included in the article's Creative Commons licence and your intended use is not permitted by statutory regulation or exceeds the permitted use, you will need to obtain permission directly from the copyright holder. To view a copy of this licence, visit http://creativecommons.org/licenses/by/4.0/. The Creative Commons Public Domain Dedication waiver (http://creativeco mmons.org/publicdomain/zero/1.0/) applies to the data made available in this article, unless otherwise stated in a credit line to the data. 
of the disease [3], with knee being the primary joint of interest $[4,5]$. Reducing pain and decreasing the progression of joint damage in patients with knee OA (KOA) is still a challenging task [6]. Early detection and assessment of KOA prognostic factors are crucial for developing management and treatments that aim at preventing irreversible damage to the knee joint leading to arthroplasty. Therefore, one of the primary goals of imaging biomarkers is to identify patients at high risk of KOA progression [7].

Trabecular bone texture analysis (TBTA) involves the examination of the vertical and horizontal trabeculae of a predefined bone region of interest (ROI). Previous studies have reported that TBTA is able to predict not only the incidence of radiographic OA [8-10] but also the radiographic progression of the disease $[8,11-15]$.

TBTA has been identified as an imaging biomarker that provides information on trabecular bone changes due to KOA. Consequently, it is important to conduct a comprehensive review that would permit a better understanding of this unfamiliar image analysis technique in the area of KOA research. The aims of this article are twofold: to provide (i) a review of the studies conducted on radiographic KOA using TBTA, and (ii) a viewpoint on future research priorities.

\section{Methods}

This review highlights original research articles published between June 1991 (publication date of the seminal paper by [16]) and March 2020, on traditional and fractal-based texture analysis of OA-related changes in trabecular bone. The SANRA criteria were used as a framework of quality assurance of the present manuscript [17]. We narrowed our focus down as follows:

- Site investigated: knee

- Imaging modality: conventional radiography

- Image processing method: texture analysis

- Target subject: human

\section{Search strategy}

Our search strategy is illustrated in Fig. 1. Literature searches were performed in the PubMed electronic database via MeSH with the publication date set between June 1st, 1991, and March 31st, 2020. The search keywords and Medical Subject Headings used were:

("Osteoarthritis"[Mesh] OR Osteoarthriti") AND ("Osteoarthritis, Knee"[Mesh] OR "Knee"[Mesh] OR "Knee Joint"[Mesh] OR "Knee Prosthesis"[Mesh] OR Knee) AND ("Radiography"[Mesh] OR "X-Rays"[Mesh] OR Radiograph* OR Xray* OR X-Ray*) AND

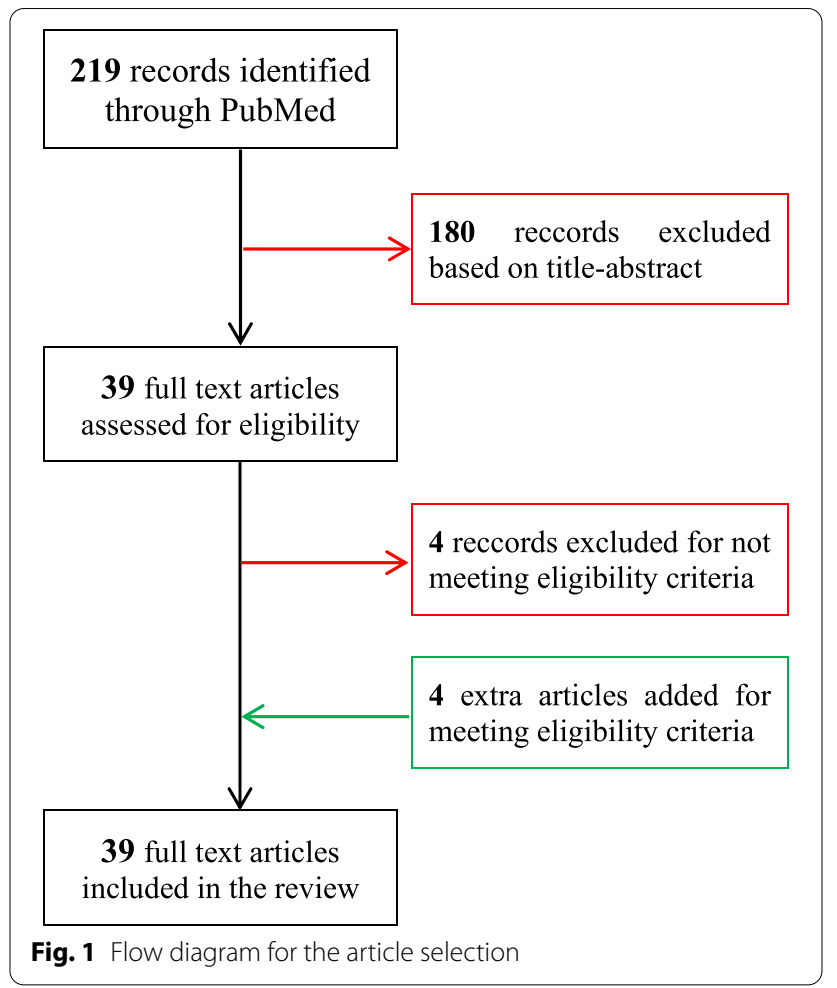

("Cancellous Bone"[Mesh] OR Trabecula* OR TBT OR Cancellous Bone) AND (“"1991/06/01”[PDat]: “2020/03/31"[PDat]) AND (English[lang])).

The reference lists of all the included studies were screened to identify any relevant missing studies and discard the wrongly selected ones. The search was limited to humans and English language full-text.

The PubMed database search resulted in 219 papers. After scanning the titles and abstracts in Zotero, 39 papers were used for further investigation.

\section{Eligibility criteria and selection process}

Articles were considered eligible if they presented quantitative work on TBTA of KOA using conventional radiography. Exclusion was not applied to statistical methods. Exclusion criteria included studies related to other than human KOA, imaging techniques other than conventional radiography, and studies without TBTA. Non-peer-reviewed articles, dissertations, abstracts, conference proceedings, commentaries, and letters to the editor were also excluded.

Two authors (A.A., E.L.) performed the selection process independently. Agreement was achieved by discussion. The full texts of potential articles were then retrieved and screened using the same procedure. 


\section{Results}

\section{Study selection}

The search strategy identified 219 studies. Based on title or abstract screening, 180 studies were excluded since they did not meet the eligibility criteria. After full-text screening, an additional 4 studies were excluded. The subsequent full-text assessment resulted in 35 records that were found eligible for the comprehensive review. An additional 4 records were identified by checking the references and were included in the current review.

\section{Data extraction}

Two authors (A.A., E.L.) independently classified the selected studies. Any disagreements between the reviewers were resolved by a consensus meeting with the third author (K.N.). The five criteria used in the classification process were (see Table 1 for the list of studies included in each category):

1. Cross-sectional quantitative evaluation between OA and non-OA knees

2. Understanding the microarchitecture of subchondral bone

3. Prediction of OA progression

4. Prediction of radiographic OA incidence

5. The association of TBTA with other endpoints

\section{What is known about TBTA and KOA Cross-sectional evaluation of changes in trabecular bone texture (TBT) between $O A$ and non-OA knees}

Several methods have been proposed for the analysis of TBT aimed at discriminating knees with and without radiographic OA. In a seminal paper published in June 1991, Lynch et al. [16]introduced a new method based on fractal dimension (FD) and applied it to digitized images of knee macroradiographs. They demonstrated that subchondral tibial TBT on these images was fractal above a certain resolution [16]. They also demonstrated that the fractal signature (FS), a numerical measure of image texture that takes into account the influence of image resolution on the $\mathrm{FD}$, was able to quantify $\mathrm{OA}$ changes in subchondral tibial bone texture roughness and directionality [25]. After further exploring the quantitative changes in subchondral trabecular bone associated with KOA in the medial compartment $[19,20]$, a larger study enrolling 110 patients with KOA compared to 27 nonOA volunteers was conducted [26]. In this study, it was reported that the trabecular bone structure quantified using FS analysis (FSA [25]) was significantly different both in the medial and lateral compartments compared to the non-OA control group [26]. The key finding was an increased FS of vertical trabeculae in OA, consistent with an "osteoporosis like" change at the subchondral ROI [26]. The development of FSA was carried out on high resolution macroradiographs. A further study was therefore conducted to show that the analysis is able to produce relevant discriminant results between $\mathrm{OA}$ and non-OA patients on conventional low-resolution radiographs [29]. It was confirmed, though in a small sample of OA $(n=24)$ and non-OA $(n=10)$ patients, that FSA could also detect significant disease-related changes in subchondral bone microarchitecture using the standard lower resolution radiographs [29]. The potential link between osteophytes and juxta-articular bone was explored by means of FSA in a cross-sectional study of 60 patients with KOA and 21 healthy non-OA volunteers [28]. The findings that emerged from this study suggest that the architectural organization of trabecular bone in the marginal regions is related to the size of the osteophytes [28].

The performance of a modified Hurst orientation transform (HOT) method, originally proposed by Podsiadlo et al. [47], was evaluated under different imaging conditions [32]. It was found that the method was sensitive to image noise, blur, and magnification, but relatively unaffected by projection angle and exposure. Three other methods (fractal signature Hurst orientation transform (FSHOT), variance orientation transform (VOT), and blanket with rotating grid (BRG)), were developed to calculate FS in all possible dimensions [40]. The objective was to overcome the limitation of the FSA and HOT methods that are able to calculate FS only in the vertical and horizontal directions. The three FS methods were

Table 1 List of studies included in the 6 categories

\begin{tabular}{|c|c|c|}
\hline$N^{\circ}$ & Category & Reference listing \\
\hline 1 & Cross-sectional quantitative evaluation between OA and non-OA knees ( 24 articles) & {$[16,18-40]$} \\
\hline 2 & Understanding the microarchitecture of subchondral bone (2 articles) & {$[41,42]$} \\
\hline 3 & Prediction of OA progression (8 articles) & {$[8,11-15,43,44]$} \\
\hline 4 & Prediction of radiographic OA incidence (3 articles) & {$[8-10]$} \\
\hline 5 & The association of TBTA with other endpoints ( 2 articles) & {$[45,46]$} \\
\hline
\end{tabular}


evaluated using computer-generated isotropic fractal surface images with known FDs and X-ray images of a human tibial head. Results showed that, overall, the VOT method was more accurate in measuring bone texture roughness and anisotropy, and also less sensitive to imaging conditions and translation of bone region than the other two methods. The VOT method was also evaluated in the determination of differences in TBT between OA and non-OA knees $[31,39]$. The study by Wolski et al. conducted on a small-size population of 46 individuals (26 controls (without OA) and 26 cases (Kellgren \& Lawrence $(K L)$ grade $\geq 2$ ) matched by sex, age, body mass index (BMI), and compartment) showed that the FD of cases was lower than that of the controls. The study also showed that, unlike HOT, the VOT method detected changes in texture roughness over a wide range of trabecular sizes and along the roughest part of the tibial bone and texture anisotropy at individual trabecular sizes. Furthermore, the VOT method was used to detect differences in TBT between patients without KOA (KL grade less than 2) but with and without cartilage defects on a different and slightly larger cohort of 48 individuals [38]. Cartilage defects were graded on MRI images using a 5-point scale in relation to the thickness of the cartilage and the level of exposure of subchondral bone. At trabecular sizes of $0.3-0.4 \mathrm{~mm}$, the results showed that in both medial and lateral compartments values of FS calculated in the vertical $\left(\mathrm{FS}_{\mathrm{V}}\right)$ and horizontal $\left(\mathrm{FS}_{\mathrm{H}}\right)$ directions were higher for cases as compared to controls. An alternative method was proposed based on distances between images quantified by a signature dissimilarity measure (SDM) [36]. The method was found to be invariant to image exposure, magnification, noise, and image size ( $>64 \times 64$ pixels). However, it was sensitive to small image sizes, projection angle, and anisotropy. The performance of the SDM method was evaluated in the detection of OA between 17 healthy and 37 OA subjects. A classification accuracy of $78.8 \%$ was achieved by the SDM method.

While the research group of Podsiadlo et al. [32, 37-40] focused mainly on fractal-based techniques, the research group of Saarakkala et al. [21-24] proposed non-fractalbased techniques. The ability of radiography-based bone texture analysis to separate patients with different $\mathrm{KL}$ grades (1-4) with and without articular cartilage damage from those with and without bone marrow lesions was evaluated in the medial subchondral bone tibia [23]. Although the subjects were symptomatic and controls could have OA-related changes, TBTA was able to discriminate the cartilage damage from BML. Non-fractalbased techniques for texture analysis have also been used to discriminate patients with different $\mathrm{KL}$ grading scores. In [24], predefined parameters obtained from both
Laplacian-based and Local Binary Pattern (LBP)-based images were studied and shown to be able to separate subjects in two groups according to their KL scores. The LBP method performed better.

Further studies have demonstrated that TBT parameters, measured in plain radiographs from KOA patients, are related to cartilage composition assessed by MRI. In [22], a weak relationship between TBT parameters selected in the medial subchondral bone and $\mathrm{T} 2$ relaxation time values of the medial tibial cartilage was found. However, no difference was observed between tibial cartilage composition and bone structural parameters in the medial tibial bone. In a sample of 109 subjects, Hirvasniemi et al. evaluated the importance of post-processing algorithms in the assessment of bone density and texture on plain radiographs [21]. The study confirmed the mandatory use of calibrated grayscale values to measure bone density. It was reported that machine learning plays an important role in exploring clinical covariates and bone density and texture, with the aim of building models that discriminate efficiently between patients with or without KOA [21].

Several methods have been proposed to demonstrate the sensitivity of TBT changes induced by treatments or other factors modifying the progression of KOA. Roemer et al. [33] studied the behavior of the trabecular bone structure extracted from radiographs of subjects exposed to increased joint point loading such as that experienced by young active athletes. Associations of FSs calculated on bone texture in the horizontal and vertical directions with four risk factors, namely athletes, gender, ACL surgery, and age, were examined. The study included 685 patients of whom 19.7\% were athletes, $81.2 \%$ were male, and $8.8 \%$ had previous ACL. Mean age was 28.5 years $(\mathrm{SD} \pm 6.5)$. Although significant differences in FSs in both compartments and in both the vertical and horizontal directions were observed, the study included a heterogeneous population, as acknowledged by the authors. Anterior cruciate ligament (ACL) injury may contribute to the development of a post-traumatic OA (PTOA) [48]. In a study of a small sample of 19 patients with ACL rupture, the radiographic changes in association with joint space width (JSW) were examined. Macro-radiographs of the patients' knees (the diseased one and the uninjured one) were acquired at a mean time from injury of 34.3 (95\% confidence interval (26.9 to 41.7) months [18]. Although no statistical difference in JSW or in subchondral cortical plate thickness was observed, there was a significant decrease in FD for ACL ruptured knees in the horizontal trabeculae of the medial compartment compared to the uninjured ones. To the best of our knowledge, the ability of these microarchitectural 
changes, detected about 4 years after injury, to predict the ongoing incidence of PTOA has not yet been evaluated. The trabecular orientation in the distal and proximal tibia was investigated by Sampath et al. [34], considering 90 OA patients, mostly with varus alignment. Significant correlations between the mechanical axis (leg alignment), calculated as the angle between the femoral and tibial axes, and trabecular orientation, determined using structure tensor-based analysis, in both distal and proximal tibia, were observed. In this study, interestingly, the trabecular anisotropy was observed to be greater in the medial tibiofemoral compartment than in the lateral side, consistent with the type of studied cases, where tibiofemoral KOA is generally more commonly observed in the medial than in the lateral compartment. In a study including 9 patients with symptomatic KOA, Miller et al. [30] investigated the differences in TBT between knees with and without a treatment based on a minimally invasive joint unloading implant. Significant changes in vertically oriented trabeculae, as reflected by FSA, were observed in this 2-year study. Results demonstrated the relationship between joint loading and subchondral trabecular structure and orientation, particularly in the medial subchondral compartment. In a recent study, the VOT method was used to evaluate changes in TBT after arthroscopic partial meniscectomy (APM) in non-radiographic OA knees (KL scores less than 2 in either knee) [37]. This study included 21 subjects (aged 35-55 years). Radiographs were acquired before APM and one year after surgery. The primary result was an increase in the ratio of medial-to-lateral TBT roughness in the knees undergoing APM as compared to the non-injured knees, before and after APM. These results might be useful in the prediction of KOA. A previous study evidenced that arthroscopic APM is an important factor of KOA risk for patients over 40 years old [49].

Periarticular and subchondral bone mineral density (BMD) may reflect a complex cross-talk between articular cartilage and subchondral bone [50] and could predict KOA progression [51]. In a pilot study of $11 \mathrm{KOA}$ and 11 non-KOA tibiae, differences in trabecular bone organization and BMD between site-matched ROIs were analyzed [27]. No differences in BMD were found. Trabecular organization measured by FSA was significantly different in OA as compared to non-OA tibiae. This indicated that TBT was more sensitive than BMD to OA changes in bone features [27]. Periprosthetic dual-energy $\mathrm{X}$-ray absorptiometry has been proposed to assess bone structural changes occurring after total knee arthroplasty (TKA) [52]. Moreover, FSA of macroradiographs of knees with total condylar knee prosthesis fixed with cement might quantify the longitudinal trabecular bone changes related to total knee arthroplasty. The trabecular changes were much greater in the lateral than in the medial compartment [53].

\section{D-3D correlation of TBT parameters}

The texture analysis of bone microarchitecture is based on plain knee radiographs, which are $2 \mathrm{D}$ projections of a 3D microstructure of trabecular bone. Consequently, the third dimension is lost and this may create ambiguities in analyzing X-ray images in spatial terms. Nevertheless, it has been reported that the textural bone parameters were associated significantly with 3D trabecular bone parameters at various bone sites including femur [42] and calcaneus $[54,55]$. These studies, however, focused mainly on osteoporosis-related changes and not on OAinduced changes. Thus, Hirvasniemi et al. investigated relationships between 2 and 3D bone structure parameters calculated for the site-matched ROIs of subchondral bone selected on plain radiographs and 3D $\mu \mathrm{CTs}$ of the proximal tibia respectively [41]. Despite a lack of any diagnosed joint disease of the cadaver knees and a lack of usual soft tissue surrounding the knee during the radiographic process, 2D bone structure parameters correlated significantly with the $3 \mathrm{D} \mu \mathrm{CT}$ parameter. There were strong inverse correlations between the FD calculated for vertical structure and the trabecular spacing [41].

\section{Prediction of radiographic $O A$ progression}

The ability of TBTA to predict the progression of KOA has been evaluated in several papers [8, 11-15, 43, 44]. The main studies are summarized in Table 2 and they show that FSA is the most popular method.

The usual clinical covariates of interest used to select patients with progressive OA include age, gender, and BMI. JSW and semi-quantitative KL grading are also commonly used in clinical trials (risedronate [46, 56], SEKOIA [57]). Fractal and non-fractal-based texture analysis methods can play an important role in the selection of patients as they provide the numerical measures of OA-induced bone changes.

In a 2-year longitudinal study conducted in 40 patients, FSA was used to measure prospectively structural bone changes observed in the subchondral and subarticular regions of their diseased proximal tibia. The main finding was the decrease in the FD of both horizontal and vertical trabeculae in OA knees compared to the non-OA ones [12]. It was also found, however, that knees with slow or detectable joint space narrowing (JSN) progression could not be separated. This is probably due to the limited size of the patient sample.

Based on a group of 30 subjects with advanced KOA (17 men and 13 women) [44], using TBT parameters, a 
Table 2 Summary of the main studies related to the prediction of OA progression

\begin{tabular}{|c|c|c|c|c|c|}
\hline & $\begin{array}{l}\text { Cohort name } \\
(m, n)\end{array}$ & Period & Definition & Progression & Major findings \\
\hline Messent et al. 2005 [12] & $\begin{array}{l}\text { King's College London } \\
(\mathrm{RG}, 40)\end{array}$ & 23 & $K L \geq 2$ & $\triangle J S N_{M} \geq 1$ & $\begin{array}{l}\text { No link was found between the degree of subchon- } \\
\text { dral trabecular bone changes, analyzed by fractal } \\
\text { signature analysis (FSA), and the rate of cartilage } \\
\text { destruction, quantified by the annual rate of joint } \\
\text { space narrowing (JSN) }\end{array}$ \\
\hline Kraus et al. 2009 [14] & $\begin{array}{l}\text { POP study } \\
(C R, 123)\end{array}$ & 36 & $1 \leq K L \leq 3$ & $\triangle J S N_{M} \geq 1$ & $\begin{array}{l}\text { Baseline trabecular bone texture analysis (TBTA), } \\
\text { measured by FSA, of the medial tibial plateau } \\
\text { was predictive of medial knee JSN progression. } \\
\text { The predictive model with maximum effective- } \\
\text { ness combined FSA, knee alignment, traditional } \\
\text { covariates, and bone mineral content at baseline } \\
\text { (AUC 0.79) }\end{array}$ \\
\hline Woloszynski et al. 2012 [43] & $\begin{array}{l}\text { University of } \\
\text { Western Australia } \\
(\mathrm{CR}, 50)\end{array}$ & 48 & $\mathrm{KL} \geq 2$ & $\triangle J S N_{M} \geq 1$ & $\begin{array}{l}\text { Baseline TBTA, measured by the dissimilarity-based } \\
\text { multiple classifier }(\mathrm{DMC}) \text {, of the tibial plateau } \\
\text { was predictive of knee JSN progression (accu- } \\
\text { racy }=0.80 \text {, specificity }=0.82 \text {, and sensitivity }=0.78 \text { ) }\end{array}$ \\
\hline Woloszynski et al. 2012 [8] & $\begin{array}{l}\text { Lund University } \\
(\mathrm{CR}, 105)\end{array}$ & 48 & $\mathrm{KL} \geq 2$ & $\triangle J S N_{M} \geq 1$ & $\begin{array}{l}\text { Baseline TBTA, measured by the signature dissimilar- } \\
\text { ity measure method (SDM), of the tibial plateau } \\
\text { was predictive of medial knee JSN progression } \\
(A \cup C=0.77)\end{array}$ \\
\hline Kraus et al. 2013 [13] & $\begin{array}{l}\text { Pfizer study } \\
(\mathrm{CR}, 58)\end{array}$ & $12-24$ & $\begin{array}{l}2 \leq \mathrm{KL} \leq 3 \\
\mathrm{JSW} \mathrm{M}_{\mathrm{M}} \geq 2 \mathrm{~mm}\end{array}$ & $\begin{array}{l}\triangle J S W_{M} \geq 5 \% \\
\triangle J S A_{M} \geq 5 \%\end{array}$ & $\begin{array}{l}\text { Bone trabecular integrity }(\mathrm{BTI}) \text {, measured by FSA, of } \\
\text { the medial tibial plateau at baseline was predictive } \\
\text { of } 5 \% \text { change in OA progression of joint space area } \\
\text { (JSA) (AUC = 0.85) }\end{array}$ \\
\hline Janvier et al. 2017 [11] & $\begin{array}{l}\text { OAl } \\
(\mathrm{CR}, 1124)\end{array}$ & 48 & $2 \leq K L \leq 3$ & $\triangle J S N_{M} \geq 1$ & $\begin{array}{l}\text { Baseline TBTA of the tibial plateau at baseline was } \\
\text { predictive of medial knee JSN progression. Best } \\
\text { model included clinical covariates, JSN and TBTA, } \\
\text { measured by Whittle estimator (Whe), (AUC }=0.75 \text { ) }\end{array}$ \\
\hline Kraus et al. 2018 [15] & $\begin{array}{l}\text { FNIH } \\
\text { (CR\&RG, 579) }\end{array}$ & $24-48$ & $\begin{array}{l}1 \leq \mathrm{KL} \leq 3 \\
\mathrm{JSN}_{\mathrm{L}}<2\end{array}$ & $\triangle J S W \_\min \geq 0.7$ & $\begin{array}{l}\text { Using baseline TBTA, medial minimum joint space } \\
\text { width (JSW) progression was characterized by thin- } \\
\text { ner vertical and thicker horizontal trabeculae (best } \\
\text { C-statistic }=0.649 \text { ) }\end{array}$ \\
\hline
\end{tabular}

$n$ total number of included subjects, $m$ image acquisition modality, $P O P$ prediction of osteoarthritis

quantification of subchondral tibial sclerosis in the setting of KOA progression was proposed. It was found that a higher grade of JSN was associated with a lower porosity and a higher number of free trabecular ends. BMI, gender, age, and knee alignment angle had strong effects on this association. However, no apparent correlations were found between TBT parameters and knee alignment angle.

High reproducibility in quantifying bone sclerosis using Tb.Sp and its significant relationship with JSW demonstrated potential for assessing OA progression.

In a cohort of 138 participants, it was reported that the model based on baseline TBT parameters outperformed those that included only JSW and traditional clinical covariates [14]. In another cohort of 60 subjects, the predictive power of TBT parameters calculated by FSA was studied [13]. KOA progression was evaluated in this 24-month longitudinal study using high-quality digital radiographs obtained from a clinical trial. As determined by both MRI and plain radiography outcomes, baseline trabecular bone parameters were able to assist in the prediction of OA progression [13].

In a relatively large population including $194 \mathrm{KOA}$ cases with both pain and radiographic progression and 406 comparators (knees without OA), the authors concluded that TBT parameters could be useful as an OA trial enrichment strategy targeting patients at high risk of disease progression [15].

The ability of TBT to predict KOA progression was also evaluated using the OAI dataset [11]. In this large population including 1124 patients, a few novelties were proposed. First, a patchwork of ROIs was proposed that covered both the medial and lateral subchondral bone regions. Second, TBTA was performed using three different methods: FSA, quadratic variations (Var) and Whittle (Whe). Third, a sensitivity analysis was conducted to evaluate the potential effect of center (X-ray devices) on the predictive capacity of TBT. Their predictive model included the clinical covariates (age, gender, BMI), JSN, and TBT parameters with an area under the ROC curve (AUC) of 0.77. The AUC is usually used to indicate the 
overall accuracy of a test according to its sensitivity and its specificity (higher accuracy as the value approaches 1 ).

In another study, Woloszynski et al. [43] proposed a dissimilarity-based multiple classifier (DMC) system that uses distances between X-ray images and a diverse classifier ensemble. This system enables prediction of KOA without the calculation and selection of TBT features. Based on a sample of 50 subjects, the system predicted KOA progression defined as an increase in the sum of JSN and osteophytes over 4 years with an accuracy of $80 \%$ [43].

For OA progression, all the studies reviewed included patients with $\mathrm{KL}=2$ and $\mathrm{KL}=3$ at baseline. Some of them also included patients with $\mathrm{KL}=1$ or $\mathrm{KL}=4$ at baseline. In terms of the TBTA method, FSA was the most widely used method.

\section{Prediction of radiographic $O A$ incidence}

The task of automatically assessing KOA severity can be approached as an image classification problem [35]. For example, an algorithm, called Weighted Neighbour Distance using a Compound Hierarchy of algorithms Representing Morphology (WND-CHRM) [35], was designed to identify biometrics that are capable of predicting $\mathrm{KOA}$ in knees without radiographic OA at baseline. The WNDCHRM generates a set of 34 descriptors from Tamura [58] and Haralick [59] texture features. In 123 pairs of $\mathrm{X}$-ray knee images, changes from normal (KL grade 0$)$ to moderate OA (KL grade 3) and minimal OA (KL grade 2) were predicted with an accuracy of $72 \%$ and $62.4 \%$, respectively. The most predictive bone regions were identified in locations adjacent to the tibial spines.

The performance of another model, based on TBTA, for predicting the incidence of radiographic KOA was examined [8]. In this 4-year follow-up study, TBT regions selected in medial and lateral compartments were analyzed in a cohort of 203 radiographic knees, using the SDM-based roughness, degree of anisotropy, and direction of anisotropy parameters. Results from this study illustrated the ability of TBTA to predict increased medial JSN in knees with or without radiographically visible KOA at baseline. The AUC was 0.77 and 0.75 for knees with and without radiographic OA, respectively, in the medial compartment. Values of the AUC were 0.71 and 0.72 in the lateral compartment. These results were adjusted according to age, sex, and BMI [8].

In a multicentre study, the relations of TBT with JSN increase and incident radiographic KOA were examined using the VOT method [9]. Analyzing a large dataset of 1433 subjects from the Multicenter Osteoarthritis Study (MOST) [60], where most of the OA incidence and the increases in JSN occurred in the medial compartment, the results showed that a higher medial bone roughness was associated with noticeable discrimination of healthy and incident OA patients at 48- and 60-month followups. The numbers of statistically significant associations found were higher for JSN increase as compared to incident OA, particularly at 30- and 60-month follow-ups. In this large dataset, TBT regions were selected using an automated method [61].

Using again the well-phenotyped population of the OAI database, it was demonstrated that TBT parameters could predict the incidence of radiographic KOA [10]. Exploiting the whole tibial proximal trabecular bone, several prediction scenarios were studied including the onset of JSN, of tibial osteophytes and the usual KL scale. Based on AUC as a global performance criterion of prediction, the inclusion of TBT parameters in the models provided AUC that ranged from 0.69 to 0.73 . In addition, diagnostic odds ratios computed to examine the relevance of the models yielded high values, indicating that TBTA could identify a subset of true OA initiators with a very low number of false positives [10].

For OA incidence, all the studies reviewed here included patients with $\mathrm{KL}=0$ at baseline. However, the group of Podsiadlo also included patients with $\mathrm{KL}=1$. In terms of the TBTA method, there was no common agreement. Surprisingly, the FSA has never been employed in this regard.

The major studies related to the prediction of OA incidence are summarized in Table 3.

\section{Association of TBTA with other endpoints (prediction of knee joint replacement and responses to pharmacological treatment)}

Besides KOA incidence and progression, the association of TBTA with other endpoints has also been investigated. In this regard, only two studies were detected in this review.

In the first study, published in 2014, the VOT method was employed to examine the association between baseline TBT and the risk of Knee Joint Replacement (KJR) over 6 years [45] using a sample of 114 subjects. Exclusion criteria included age of less than 40 years, WOMAC score of less than $20 \%$, osteophyte grade of 0 , KL grade of 4 , or inadequate quality of the digitized radiographs for fractal analysis. If both knees were symptomatic, the knee with more severe change was excluded. The study showed that knees with a lower TBT mean FD (FD mean) at baseline had an increased risk of KJR, independent of age, gender, BMI, grade of osteophyte and JSN. This may provide a target for novel intervention strategies in this condition.

In the second study, published in 2007, the association of TBTA with treatment responses of KOA patients was evaluated [46]. 
Table 3 Summary of the major studies related to the prediction of OA radiographic incidence

\begin{tabular}{|c|c|c|c|c|c|}
\hline & $\begin{array}{l}\text { Cohort name } \\
(m, n)\end{array}$ & Period & Definition & Incidence & Major findings \\
\hline Shamir et al. 2009 [35] & $\begin{array}{l}\text { BLSA } \\
\text { (Unknown, 123) }\end{array}$ & 240 & $\mathrm{KL}=0$ & $\Delta K L \geq 2$ & $\begin{array}{l}\text { Baseline TBTA, using a Compound Hierarchy of Algorithms Represent- } \\
\text { ing Morphology (WND-CHARM) algorithm, of the region adjacent } \\
\text { to the tibial spines was predictive of knee Kellgren-Lawrence (KL) } \\
\text { incidence (best accuracy }=0.72, \mathrm{KL} \text { from } 0 \text { to } 3 \text { ) }\end{array}$ \\
\hline Woloszynski et al. 2012 [8] & $\begin{array}{l}\text { Lund University } \\
(C R, 105)\end{array}$ & 48 & $\mathrm{KL}=0$ & $\triangle J S N_{M} \geq 1$ & $\begin{array}{l}\text { Baseline TBTA, measured by the signature dissimilarity measure } \\
\text { method (SDM), of the tibial plateau was predictive of medial knee } \\
\text { JSN incidence (AUC }=0.75 \text { ) }\end{array}$ \\
\hline Podsiadlo et al. 2016 [9] & $\begin{array}{l}\text { MOST } \\
\text { (CR\&RG, 1433) }\end{array}$ & $48-60$ & $\mathrm{KL} \leq 1$ & $\Delta J S N_{M} \geq 1$ & $\begin{array}{l}\text { Baseline TBTA, measured by the variance orientation transform (VOT) } \\
\text { method, was associated with incident radiographic OA, indepen- } \\
\text { dently of risk factors for knee OA. Most of the OA incidence occurred } \\
\text { in medial compartments }\end{array}$ \\
\hline Janvier et al. 2017 [10] & $\begin{array}{l}\text { OAl } \\
(C R, 319)\end{array}$ & 48 & $\mathrm{KL}=0$ & $\Delta J S N_{M} \geq 1$ & $\begin{array}{l}\text { Baseline TBTA of the tibial plateau at baseline was predictive of medial } \\
\text { knee JSN progression. The best model included TBTA, measured by } \\
\text { the quadratic Variations estimator ( } \operatorname{Var})(A \cup C=0.73)\end{array}$ \\
\hline
\end{tabular}

$n$ total number of included subjects, $m$ image acquisition modality, BLSA Baltimore Longitudinal Study of Aging

To examine whether risedronate (an antiresorptive agent) could slow down or halt progressive JSN in OA patients, a double-blind randomized placebo-controlled study was conducted using subjects with KOA [56]. Within the framework of this study, another 2-year longitudinal radiographic study examining the effect of risedronate on subchondral bone loss in KOA patients was published, in which FSA was used to quantify longitudinal changes separately in horizontal and vertical trabeculae in ROIs selected in the medial subchondral compartment [46]. TBTA in the trial demonstrated a dose-dependent therapeutic drug effect characterized by retention of normal trabecular structure in the knee of progressors with JSN [46]. These results, whatever the clinical relevance of the use of boneacting agents in $\mathrm{KOA}$, illustrate the responsiveness of texture parameters to pharmacological treatment.

TBTA could provide markers for the enrichment of clinical trials and thereby help to enhance the screening of OA progressors and reduce the screen failure rates [15].

\section{Perspectives (What is unknown or should be known)}

Towards an "optimal" procedure for bone texture analysis The above review shows that several aspects related to TBT analyses of knee radiographs have already been investigated and validated. These are summarized in this chapter and Table 4.

First, TBT changes constitute one of the integral features of $\mathrm{OA}$ initiation and progression. In this regard, different methods have been proposed and evaluated in the analysis of TBT of OA patients. These activities yielded a large number of texture descriptors without a clear group of the best performers [21], as shown in Table 5 .

Second, it has been demonstrated that the TBT phenotype of patients with progressive $\mathrm{KOA}$ is associated with apparent thickening of horizontal trabeculae and thinning of vertical trabeculae $[15,38]$.

Third, TBT parameters evaluated on radiographs correlate significantly with the subchondral trabecular microarchitecture assessed by $\mu \mathrm{CT}$ [41].

Fourth, when considering TBT parameters in OA trials, predictive models [11] may perform well without the need for supplementary procedures for the cross-calibration of devices at different clinical centers as standardized radiographic data acquisition procedures were usually performed in phase III trials $[11,60]$.

It would be of great interest to the OA research community to conduct a longitudinal study, based on a large dataset (e.g., OAI or MOST cohorts), in order to compare the four main fractal-based methods (FSA [25], Var

Table 4 TBT on radiographs in KOA: what is already known

\begin{tabular}{ll}
\hline $\boldsymbol{N}^{\mathbf{0}}$ & What is already known \\
\hline 1 & TBT changes constitute one of the integral features of OA initiation and progression \\
2 & TBT phenotype of patients with progressive OA is associated with apparent thickening of horizontal trabeculae and thinning \\
Of vertical trabeculae & TBT parameters evaluated from 2D radiographs reflect the subchondral trabecular bone 3D microarchitecture assessed by CT \\
4 & $\begin{array}{l}\text { Using TBT parameters in OA trials does not require supplementary methods for cross-calibration of devices at different clini- } \\
\text { Cal centers in addition to the standardized radiographic data acquisition procedures already performed in phase III trials }\end{array}$ \\
\hline
\end{tabular}


Table 5 Best TBT descriptors from selected studies, for different OA-related tasks

\begin{tabular}{|c|c|c|c|}
\hline & Population & Major findings & Best descriptor \\
\hline Messent et al. (2005) [26] & 110 & $\begin{array}{l}\text { FSA of vertical FSA was greater in OA subjects than non-OA sub- } \\
\text { jects, consistent with increased vertical trabecular number associ- } \\
\text { ated with thinning and fenestration of coarser trabeculae }\end{array}$ & Increase in vertical FD \\
\hline Messent et al. (2005) [12] & 40 & $\begin{array}{l}\text { No link was found between the degree of subchondral trabecular } \\
\text { bone changes, analyzed by fractal signature analysis (FSA), and } \\
\text { the rate of cartilage destruction, quantified by the annual rate of } \\
\text { joint space narrowing (JSN) }\end{array}$ & Decrease in vertical FD and horizontal FD \\
\hline Wolski et al. 2011 [38] & 48 & $\begin{array}{l}\text { Significant differences in TBT, measured by that the variance } \\
\text { orientation transform (VOT) method, between subjects with and } \\
\text { without cartilage defects, suggesting thinning and fenestration } \\
\text { of trabecular bone in knees with cartilage defects }\end{array}$ & Increase in vertical FD and horizontal FD \\
\hline Janvier et al. (2017) [11] & 1124 & $\begin{array}{l}\text { Baseline TBTA of the tibial plateau at baseline was predictive of } \\
\text { medial knee JSN progression. The best model included clinical } \\
\text { covariates, JSN and TBTA, measured by Whittle estimator (Whe) } \\
\text { (AUC }=0.75 \text { ) }\end{array}$ & Increase in vertical FD \\
\hline
\end{tabular}

[62], VOT [40], and Whe [63]) as well as other published TBT descriptors in order to examine the performance of these different parameters, separately or in a composite way. The prediction of OA progression or incidence has been evaluated in subsets of the two large OA cohorts (OAI and MOST) using digitized films and computed radiography. To the best of our knowledge, the effect of the modality type on the performance of prediction models has not yet been evaluated when using the different TBTA methods, with the exception of the Var method [64]. The choice of imaging modality is an important decision in routine clinical practice for diagnosis of KOA [65].

Furthermore, the selection of radiographs eligible for TBTA has not been addressed either. In the OAI cohort, several indicators are used to describe the quality of the radiographs, such as alignment, positioning, exposure problems, and visible materials and artifacts. These indicators however are not sufficient to select ROIs with the accuracy necessary for TBTA. Furthermore, in the MOST cohort, no information is included to describe the quality of radiographs (over/underexposed or with loss of details in the ROI zones). Researchers are therefore encouraged to provide sub-datasets of OAI and MOST that can be used as a gold standard for KOA progression and incidence. As examples, we suggest considering the FNIH subset proposed by Kraus et al. [15] for the prediction of OA progression in the OAI cohort, and the subset proposed by Podsiadlo et al. [9] in MOST and by Janvier et al. [10] in OAI, for the prediction of OA incidence, because of the automated procedures and the locations of bone regions.

In order to ensure the selection of radiographs on which TBTA is possible, adding a quality-control flag to
OAI and MOST would be of great interest to the KOA research community.

Previous studies have reported that the tibial medial to lateral $(\mathrm{M}: \mathrm{L})$ ratio of subchondral $\mathrm{BMD}$ was associated with meniscal lesions and also with bone marrow lesions $[66,67]$. These associations suggested that there is a link between load distribution and features of OA in knee joints. The M:L ratio of TBT descriptors was evaluated in non-radiographic OA knees after APM [37], indicating their potential for detecting early signs of OA development. However, this ratio is yet to be evaluated with radiographic OA knees.

TBT descriptors enhance the overall prediction of OA progression and incidence. However, their threshold values that provide a good trade-off between sensitivity and specificity in order to differentiate between progressors and non-progressors are still unknown.

As shown in Table 2, the prognostic capability of TBT versus changes in minimum JSW has been previously evaluated. However, researchers are encouraged to evaluate its capability versus changes in articular cartilage degeneration assessed by MRI [22], or over features of interest such as bone medullary lesions and synovitis.

There is growing interest in the use of deep learning (DL) techniques in medical imaging research [68]. DL has already shown great potential in the prediction of OA detection and progression. Recently, a DL-based method was used to estimate KL grades from plain radiographs, as part of a fully-automated model of OA detection $[68,69]$ and progression prediction [70]. DL-based methods have been proposed to predict the progression of radiographic joint space loss [71] and also to integrate subchondral bone texture information in the training criterion [72] to be used in the early detection of KOA. 
The combination of DL-based methods and TBTA was recently investigated in the OAI and MOST cohorts and showed promising results [64].

\section{Towards the validation of TBT as a biomarker in KOA}

As reviewed above, there are several cross-sectional case-control studies that showed statistically different values of TBT parameters between KOA cases and controls [23, 24, 29, 39]. Moreover, in longitudinal studies, it was reported that TBT parameters were associated with three endpoints in KOA, namely KOA radiographic incidence [8-10], KOA progression [8,11-15, 43, 44] or knee prosthetic surgery [45].

Taking these findings together, we now have a great body of evidence that TBT parameters are, indeed, associated and reflect alterations of the subchondral bone tissue observed in KOA. Thus, we can conclude that the biological plausibility for TBTA in KOA is already established.

Although it can be considered that TBT parameters have not been evaluated prospectively in those studies, as compared to some relevant established biological biomarkers [73], TBT parameters can be considered as imaging-based biomarkers that have been collected and acquired at the baseline and then stored and measured retrospectively in all individuals of the whole cohort, thus providing a robust assessment of the association between TBT parameters and OA endpoints studied in those cohorts (OULU [23], MOST [9], OAI [10]).

Whether TBT parameters would change consistently in response to potential OA treatment is still unclear. In [46], results obtained in terms of texture changes under the effect of risedronate could be easily compatible with the mechanism of action of an antiresorptive agent, i.e., a preservation of vertical trabecular structure in the group of patients that received risedronate $15 \mathrm{mg} /$ day, and even an increase in the vertical trabecular number in the group with a higher dose $(50 \mathrm{mg} /$ day). Such a relationship between the use of an antiosteoporotic agent with known mechanism of action on subchondral bone tissue and changes in TBT parameters, possibly with dosedependent effects, would be another important reason for considering TBTA as a useful biomarker for OA trials.

Finally, it is also conceivable that changes in TBT parameters under the effect of treatment could explain a relevant proportion of the preventive effect on KOA progression.

\section{Conclusions and research agenda}

It has been reported that the association of TBT parameters with clinical (age, sex, BMI) and radiological (KL, OARSI grades) covariates improves the prediction of
OA progression $[11,12,15]$ and incidence $[9,10]$. With the objective of providing a fully-automated prediction method, several studies focused on the automated calculation of TBT parameters and also KL [74] and JSW [75] grades. In this regard, there has been significant progress in providing computer-aided utilities for automated KOA assessment $[76,77]$.

The results of this review study confirm that TBTA is a relevant bioimaging marker in KOA. However, we need more knowledge to really assess what bone texture parameters are representative of, in terms of topologic or histomorphometric indices.

The research agenda would include a greater focus on the association of TBTA with the treatment responses of KOA patients. It would also include the evaluation of the association between baseline TBT and the risk of $\mathrm{KJR}$ using other cohorts and comparing it to the results obtained by [45]. In addition, the research agenda would involve the investigation of the performance of incidence or progression prediction models based on the TBTA of not only XR images but also their corresponding MRI images. The combination of other markers obtained from XR imaging (KL, JSW, etc.) and MRI (synovitis, bone marrow lesions, etc.) would be of interest to KOA research studies.

\section{Abbreviations \\ 2D: Two-dimensional; 3D: Three-dimensional; ACL: Anterior cruciate ligament; APM: Arthroscopic partial meniscectomy; AUC: Area under the receiver operating characteristic curve; BLSA: Baltimore Longitudinal Study of Aging; BMD: Bone mineral density; BMI: Body mass index; BRG: Blanket with rotating grid; DL: Deep learning; DMC: Dissimilarity-based Multiple Classifier; FD: Fractal dimension; FS: Fractal signature; FSA: Fractal signature analysis; FSH: Fractal dimension calculated in the horizontal direction; FSHOT: Fractal signature Hurst orientation transform; FSV: Fractal dimension calculated in the vertical direction; HOT: Hurst orientation transform; JSN: Joint space narrowing; JSW: Joint space width; KJR: Knee joint replacement; KL: Kellgren \& Lawrence; KOA: Knee osteoarthritis; LBP: Local binary pattern; M:L: Medial to lateral; MeSH: Medical Subject Headings; MOST: Multicenter Osteoarthritis Study; MRI: Mag- netic resonance imaging; OA: Osteoarthritis; OARSI: Osteoarthritis Research Society International; POP: Prediction of osteoarthritis progression; PTOA: Post- traumatic osteoarthritis; ROC: Receiver operating characteristic; ROI: Region of interest; SANRA: Scale for the quality Assessment of Narrative Review Articles; SDM: Signature dissimilarity measure; Tb.Sp: Trabecular separation; TBT: Trabecular bone texture; TBTA:Trabecular bone texture analysis; Var: Quadratic variations; VOT: Variance orientation transform; Whe: Whittle; WND-CHRM: Weighted Neighbour Distance using a Compound Hierarchy of algorithms Representing Morphology; WOMAC: Western Ontario and MAcMaster; $\mu \mathrm{CT}$ : Micro-computed tomography.}

\section{Acknowledgements \\ The authors gratefully acknowledge Dr John Lynch for fruitful discussion. The points raised with him during the 2 nd meeting of the KOPI Consortium enriched our conception and design of the article. They would also like to thank the participants and staff of the OAl and MOST studies. They gratefully acknowl- edge the STUDIUM for organizing group meetings related to this study. They also wish to thank Mrs. Elizabeth Rowley-Jolivet for proofreading the article.}

\section{Authors' contributions}

All authors contributed to the conception and design of the study. All authors were involved in drafting the article or revising it critically for important 
intellectual content, and all authors approved the final version to be published. Ahmad Almhdie-Imjabbar and Eric Lespessailles contributed, in addition, to data collection and analysis.

\section{Funding}

The study was funded by the European Regional Development Fund (ERDF)Project EX004579 and the ERDF- Project PRIMMO. Funding sources had no role in the study design, collection, analysis, and interpretation of the data or the decision to submit the manuscript for publication.

\section{Availability of data and materials}

All data generated or analyzed during this study are included in this published article.

\section{Declarations}

Ethics approval and consent to participate

Not applicable.

\section{Consent for publication}

Not applicable.

\section{Competing interests}

All authors state that they have no conflicts of interest.

\section{Author details}

${ }^{1}$ EA 4708- I3MTO Laboratory, University of Orleans, Orleans, France. ${ }^{2}$ Translational Medicine Research Platform, PRIMMO, Regional Hospital of Orleans, Orleans, France. ${ }^{3}$ Tribology Laboratory, School of Civil and Mechanical Engineering, Curtin University, Bentley, WA 6102, Australia. ${ }^{4}$ ImageBiopsy Lab, Vienna, Austria. ${ }^{5}$ Department of Rheumatology, Regional Hospital of Orleans, Orleans, France. ${ }^{6}$ Physics and Technology, Research Unit of Medical Imaging, University of Oulu, Oulu, Finland. ${ }^{7}$ Department of Diagnostic Radiology, Oulu University Hospital, Oulu, Finland.

Received: 13 April 2021 Accepted: 28 July 2021

Published online: 06 August 2021

\section{References}

1. Musumeci G, Aiello FC, Szychlinska MA, Di Rosa M, Castrogiovanni P, Mobasheri A. Osteoarthritis in the XXIst Century: Risk Factors and Behaviours that Influence Disease Onset and Progression. Int J Mol Sci. 2015;16:6093-112. Available from: https://www.ncbi.nlm.nih.gov/pmc/ articles/PMC4394521/. [cited 2020 Apr 28]

2. Hootman JM, Helmick CG, Brady TJ. A public health approach to addressing arthritis in older adults: the most common cause of disability. Am J Public Health. 2012;102:426-33.

3. Bijlsma JWJ, Berenbaum F, Lafeber FPJG. Osteoarthritis: an update with relevance for clinical practice. Lancet. 2011;377:2115-26.

4. Boesen M, Ellegaard K, Henriksen M, Gudbergsen H, Hansen P, Bliddal H, et al. Osteoarthritis year in review 2016: imaging. Osteoarthritis Cartilage. 2017;25:216-26. Available from: https://pubmed.ncbi.nlm.nih.gov/? term=Osteoarthritis\%20year\%20in\%20review\%202016:\%20imaging. [cited 2020 Apr 28]

5. Guermazi A, Hayashi D, Eckstein F, Hunter DJ, Duryea J, Roemer FW. Imaging of Osteoarthritis. Rheumatic Dis Clin North Am. 2013;39:67-105. Available from: http://www.sciencedirect.com/science/article/pii/S0889 $857 \times 12001159$

6. Glyn-Jones S, Palmer AJR, Agricola R, Price AJ, Vincent TL, Weinans $H$, et al. Osteoarthritis Lancet. 2015;386:376-87.

7. Karsdal MA, Michaelis M, Ladel C, Siebuhr AS, Bihlet AR, Andersen JR, et al. Disease-modifying treatments for osteoarthritis (DMOADs) of the knee and hip: lessons learned from failures and opportunities for the future. Osteoarthr Cartil. 2016;24:2013-21.

8. Woloszynski T, Podsiadlo P, Stachowiak GW, Kurzynski M, Lohmander LS, Englund M. Prediction of progression of radiographic knee osteoarthritis using tibial trabecular bone texture. Arthritis Rheum. 2012;64:688-95.
9. Podsiadlo P, Nevitt MC, Wolski M, Stachowiak GW, Lynch JA, Tolstykh I, et al. Baseline trabecular bone and its relation to incident radiographic knee osteoarthritis and increase in joint space narrowing score: directional fractal signature analysis in the MOST study. Osteoarthr Cartil. 2016;24:1736-44.

10. Janvier T, Jennane R, Toumi H, Lespessailles E. Subchondral tibial bone texture predicts the incidence of radiographic knee osteoarthritis: data from the Osteoarthritis Initiative. Osteoarthr Cartil. 2017;25:2047-54.

11. Janvier T, Jennane R, Valery A, Harrar K, Delplanque M, Lelong C, et al. Subchondral tibial bone texture analysis predicts knee osteoarthritis progression: data from the Osteoarthritis Initiative: Tibial bone texture \& knee OA progression. Osteoarthr Cartil. 2017;25:259-66.

12. Messent EA, Ward RJ, Tonkin CJ, Buckland-Wright C. Tibial cancellous bone changes in patients with knee osteoarthritis. A short-term longitudinal study using Fractal Signature Analysis. Osteoarthritis and Cartilage. 2005;13:463-70. Available from: https://www.oarsijournal.com/article/ S1063-4584(05)00025-7/abstract. [cited 2020 May 19]

13. Kraus VB, Feng S, Wang S, White S, Ainslie M, Graverand M-PHL, et al. Subchondral bone trabecular integrity predicts and changes concurrently with radiographic and magnetic resonance imaging-determined knee osteoarthritis progression. Arthritis Rheum. 2013;65:1812-21.

14. Kraus VB, Feng $S$, Wang $S$, White $S$, Ainslie M, Brett A, et al. Trabecular morphometry by fractal signature analysis is a novel marker of osteoarthritis progression. Arthritis Rheum. 2009;60:3711-22.

15. Kraus VB, Collins JE, Charles HC, Pieper CF, Whitley L, Losina E, et al. Predictive Validity of Radiographic Trabecular Bone Texture in Knee Osteoarthritis: The Osteoarthritis Research Society International/Foundation for the National Institutes of Health Osteoarthritis Biomarkers Consortium. Arthritis Rheumatol (Hoboken, NJ). 2018;70:80-7.

16. Lynch JA, Hawkes DJ, Buckland-Wright JC. Analysis of texture in macroradiographs of osteoarthritic knees using the fractal signature. Phys Med Biol. 1991;36:709-22.

17. Baethge C, Goldbeck-Wood S, Mertens S. SANRA—a scale for the quality assessment of narrative review articles. Res Integrity Peer Rev. 2019;4:5. https://doi.org/10.1186/s41073-019-0064-8.[cited2020Nov30].

18. Buckland-Wright JC, Lynch JA, Dave B. Early radiographic features in patients with anterior cruciate ligament rupture. Ann Rheum Dis. 2000;59:641-6.

19. Buckland-Wright JC, Lynch JA, Macfarlane DG. Fractal signature analysis measures cancellous bone organisation in macroradiographs of patients with knee osteoarthritis. Ann Rheum Dis. 1996;55:749-55.

20. Buckland-Wright JC, Lynch JA, Bird C. Microfocal Techniques in Quantitative Radiography: Measurement of Cancellous Bone Organization. Rheumatology. 1996;35:18-22. Available from: https://doi.org/10.1093/ rheumatology/35.suppl_3.18. [cited 2020 Dec 30]

21. Hirvasniemi J, Niinim?ki J, Thevenot J, Saarakkala S. Bone Density and Texture from Minimally Post-Processed Knee Radiographs in Subjects with Knee Osteoarthritis. Ann Biomed Eng. 2019;47:1181-90.

22. Hirvasniemi J, Thevenot J, Multanen J, Haapea M, Heinonen A, Nieminen MT, et al. Association between radiography-based subchondral bone structure and MRI-based cartilage composition in postmenopausal women with mild osteoarthritis. Osteoarthr Cartil. 2017;25:2039-46.

23. Hirvasniemi J, Thevenot J, Guermazi A, Podlipská J, Roemer FW, Nieminen MT, et al. Differences in tibial subchondral bone structure evaluated using plain radiographs between knees with and without cartilage damage or bone marrow lesions - the Oulu Knee Osteoarthritis study. Eur Radiol. 2017:27:4874-82.

24. Hirvasniemi J, Thevenot J, Immonen V, Liikavainio T, Pulkkinen P, JämsäT, et al. Quantification of differences in bone texture from plain radiographs in knees with and without osteoarthritis. Osteoarthr Cartil. 2014;22:1724-31.

25. Lynch JA, Hawkes DJ, Buckland-Wright JC. A robust and accurate method for calculating the fractal signature of texture in macroradiographs of osteoarthritic knees. Med Inform (Lond). 1991;16:241-51.

26. Messent EA, Ward RJ, Tonkin CJ, Buckland-Wright C. Cancellous bone differences between knees with early, definite and advanced joint space loss; a comparative quantitative macroradiographic study. Osteoarthr Cartil. 2005;13:39-47.

27. Messent EA, Buckland-Wright JC, Blake GM. Fractal analysis of trabecular bone in knee osteoarthritis $(\mathrm{OA})$ is a more sensitive marker of disease status than bone mineral density (BMD). Calcif Tissue Int. 2005;76:419-25. 
28. Messent EA, Ward RJ, Tonkin CJ, Buckland-Wright C. Osteophytes, juxta-articular radiolucencies and cancellous bone changes in the proximal tibia of patients with knee osteoarthritis. Osteoarthr Cartil. 2007;15:179-86.

29. Messent EA, Ward RJ, Tonkin CJ, Buckland-Wright C. Differences in trabecular structure between knees with and without osteoarthritis quantified by macro and standard radiography, respectively. Osteoarthr Cartil. 2006;14:1302-5.

30. Miller LE, Sode M, Fuerst T, Block JE. Joint unloading implant modifies subchondral bone trabecular structure in medial knee osteoarthritis: 2-year outcomes of a pilot study using fractal signature analysis. Clin Interv Aging. 2015;10:351-7.

31. Podsiadlo P, Dahl L, Englund M, Lohmander LS, Stachowiak GW. Differences in trabecular bone texture between knees with and without radiographic osteoarthritis detected by fractal methods. Osteoarthr Cartil. 2008;16:323-9.

32. Podsiadlo P, Stachowiak GW. Analysis of trabecular bone texture by modified Hurst orientation transform method. Med Phys. 2002;29:460-74.

33. Roemer FW, Jarraya M, Niu J, Duryea J, Lynch JA, Guermazi A. Knee joint subchondral bone structure alterations in active athletes: a cross-sectional case-control study. Osteoarthr Cartil. 2015;23:2184-90.

34. Sampath SA, Lewis S, Fosco M, Tigani D. Trabecular orientation in the human femur and tibia and the relationship with lower-limb alignment for patients with osteoarthritis of the knee. J Biomech. 2015;48:1214-8.

35. Shamir L, Ling SM, Scott W, Hochberg M, Ferrucci L, Goldberg IG. Early detection of radiographic knee osteoarthritis using computer-aided analysis. Osteoarthr Cartil. 2009;17:1307-12.

36. Woloszynski T, Podsiadlo P, Stachowiak GW, Kurzynski M. A signature dissimilarity measure for trabecular bone texture in knee radiographs. Med Phys. 2010;37:2030-42.

37. Wolski M, Thorlund JB, Stachowiak GW, Holsgaard-Larsen A, Creaby MW Jørgensen GM, et al. Early tibial subchondral bone texture changes after arthroscopic partial meniscectomy in knees without radiographic OA: A prospective cohort study. J Orthop Res. 2020;38(8):1819-25.

38. Wolski M, Stachowiak GW, Dempsey AR, Mills PM, Cicuttini FM, Wang Y, et al. Trabecular bone texture detected by plain radiography and variance orientation transform method is different between knees with and without cartilage defects. J Orthop Res. 2011;29:1161-7.

39. Wolski M, Podsiadlo P, Stachowiak GW, Lohmander LS, Englund M. Differences in trabecular bone texture between knees with and without radiographic osteoarthritis detected by directional fractal signature method. Osteoarthr Cartil. 2010;18:684-90

40. Wolski M, Podsiadlo P, Stachowiak GW. Directional fractal signature analysis of trabecular bone: evaluation of different methods to detect early osteoarthritis in knee radiographs. Proc Inst Mech Eng H. 2009;223:211-36.

41. Hirvasniemi J, Thevenot J, Kokkonen HT, Finnilä MA, Venäläinen MS, Jämsä T, et al. Correlation of Subchondral Bone Density and Structure from Plain Radiographs with Micro Computed Tomography Ex Vivo. Ann Biomed Eng. 2016;44:1698-709.

42. Le Corroller T, Pithioux M, Chaari F, Rosa B, Parratte S, Maurel B, et al. Bone texture analysis is correlated with three-dimensional microarchitecture and mechanical properties of trabecular bone in osteoporotic femurs. J Bone Miner Metab. 2013;31:82-8.

43. Woloszynski T, Podsiadlo P, Stachowiak GW, Kurzynski M. A dissimilaritybased multiple classifier system for trabecular bone texture in detection and prediction of progression of knee osteoarthritis. Proc Inst Mech Eng H. 2012;226:887-94.

44. Wong AKO, Beattie KA, Emond PD, Inglis D, Duryea J, Doan A, et al. Quantitative analysis of subchondral sclerosis of the tibia by bone texture parameters in knee radiographs: site-specific relationships with joint space width. Osteoarthr Cartil. 2009;17:1453-60.

45. Podsiadlo P, Cicuttini FM, Wolski M, Stachowiak GW, Wluka AE. Trabecular bone texture detected by plain radiography is associated with an increased risk of knee replacement in patients with osteoarthritis: a 6 year prospective follow up study. Osteoarthr Cartil. 2014;22:71-5.

46. Buckland-Wright JC, Messent EA, Bingham CO, Ward RJ, Tonkin C. A 2 yr longitudinal radiographic study examining the effect of a bisphosphonate (risedronate) upon subchondral bone loss in osteoarthritic knee patients. Rheumatology (Oxford). 2007;46:257-64.
47. Podsiadlo P, Stachowiak GW. Applications of Hurst orientation transform to the characterization of surface anisotropy. Tribol Int. 1999;32:387-92. Available from: http://www.sciencedirect.com/science/article/pii/S0301 679X99000584

48. Wang L-J, Zeng N, Yan Z-P, Li J-T, Ni G-X. Post-traumatic osteoarthritis following ACL injury. Arthritis Res Ther. 2020;22:57.

49. Rangger C, Klestil T, Gloetzer W, Kemmler G, Benedetto KP. Osteoarthritis after arthroscopic partial meniscectomy. Am J Sports Med. 1995;23:240-4.

50. Lespessailles E, Jennane R. Assessment of bone mineral density and radiographic texture analysis at the tibial subchondral bone. Osteoporos Int. 2012;23(Suppl 8):S871-876.

51. Lo GH, Schneider E, Driban JB, Price LL, Hunter DJ, Eaton CB, et al. Periarticular bone predicts knee osteoarthritis progression: Data from the Osteoarthritis Initiative. Semin Arthritis Rheumatism. 2018 ;48:155-61. Available from: http://www.sciencedirect.com/science/article/pii/S0049 017217300896. [cited 2020 May 6]

52. Petersen MM. Bone mineral measurements at the knee using dual photon and dual energy X-ray absorptiometry: Methodological evaluation and clinical studies focusing on adaptive bone remodeling following lower extremity fracture, total knee arthroplasty, and partial versus total meniscectomy. Acta Orthop Scand. 2000;71:1-37. https://doi.org/10. 1080/000164700753767935.[cited2020May19].

53. Papaloucas CD, Earnshaw P, Tonkin C, Buckland-Wright JC. Quantitative radiographic assessment of cancellous bone changes in the proximal tibia after total knee arthroplasty: a 3-year follow-up study. Calcif Tissue Int. 2004;74:429-36.

54. Pothuaud L, Porion P, Lespessailles E, Benhamou CL, Levitz P. A new method for three-dimensional skeleton graph analysis of porous media: application to trabecular bone microarchitecture. J Microsc. 2000:199:149-61.

55. Jennane R, Harba R, Lemineur G, Bretteil S, Estrade A, Benhamou CL. Estimation of the 3D self-similarity parameter of trabecular bone from its 2D projection. Med Image Anal. 2007;1 1:91-8. Available from: http:// www.sciencedirect.com/science/article/pii/S136184150600082X. [cited 2020 Jun 24]

56. Bingham CO, Buckland-Wright JC, Garnero P, Cohen SB, Dougados M, Adami S, et al. Risedronate decreases biochemical markers of cartilage degradation but does not decrease symptoms or slow radiographic progression in patients with medial compartment osteoarthritis of the knee: results of the two-year multinational knee osteoarthritis structural arthritis study. Arthritis Rheum. 2006;54:3494-507.

57. Reginster JY. Efficacy and safety of strontium ranelate in the treatment of knee osteoarthritis: results of a double-blind randomised, placebocontrolled trial. Ann Rheum Dis. 2014;73:e8.

58. Tamura H, Mori S, Yamawaki T. Textural Features Corresponding to Visual Perception. IEEE Trans Syst Man Cybern. 1978;8:460-73.

59. Haralick R, Shanmugam K, Dinstein I. Textural Features for Image Classification. IEEE Trans Syst Man Cybern. 1973;SMC-3:610-21.

60. Segal NA, Nevitt MC, Gross KD, Gross KD, Hietpas J, Glass NA, et al. The Multicenter Osteoarthritis Study: opportunities for rehabilitation research. PM R. 2013;5:647-54.

61. Podsiadlo P, Wolski M, Stachowiak GW. Automated selection of trabecular bone regions in knee radiographs. Med Phys. 2008;35:1870-83.

62. Istas J, Lang G. Quadratic variations and estimation of the local Hölder index of a Gaussian process. Annales de l'Institut Henri Poincare (B) Probability and Statistics [Internet]. 1997;33:407-36. Available from: http:// www.sciencedirect.com/science/article/pii/S0246020397800994. [cited 2020 Apr 12]

63. Harrar K, Hamami L, Lespessailles E, Jennane R. Piecewise Whittle estimator for trabecular bone radiograph characterization. Biomedical Signal Processing and Control [Internet]. 2013;8:657-66. Available from: http:// www.sciencedirect.com/science/article/pii/S1746809413000943. [cited 2020 Apr 12]

64. Nguyen KL, Xavier A, Almhdie A, Ibrahim N, Toumi H, Jennane R, et al. Interest of texture analysis and neural networks for the characterization of knee osteoarthritis radiographic progression in OAI and MOST cohorts. Bone Rep. 2020;13:100696. Available from: http://www.sciencedirect. com/science/article/pii/S2352187220304563. [cited 2020 Dec 28]

65. Hayashi D, Roemer FW, Guermazi A. Imaging of osteoarthritis—recent research developments and future perspective. Br J Radiol. 2018;91. 
Available from: https://www.ncbi.nlm.nih.gov/pmc/articles/PMC61 90779/. [cited 2020 Dec 31]

66. Lo GH, Hunter DJ, Nevitt M, Lynch J, McAlindon TE. Strong association of MRI meniscal derangement and bone marrow lesions in knee osteoarthritis: data from the osteoarthritis initiative. Osteoarthritis Cartilage. 2009;17:743-7. Available from: http://www.sciencedirect.com/science/ article/pii/S1063458408003610. [cited 2020 Jun 12]

67. Lowitz T, Museyko O, Bousson V, Laouisset L, Kalender WA, Laredo J-D, et al. Bone marrow lesions identified by MRI in knee osteoarthritis are associated with locally increased bone mineral density measured by QCT. Osteoarthr Cartil. 2013;21:957-64.

68. Kokkotis C, Moustakidis S, Papageorgiou E, Giakas G, Tsaopoulos DE. Machine learning in knee osteoarthritis: A review. Osteoarthritis and Cartilage Open. 2020;100069. Available from: http://www.sciencedirect. com/science/article/pii/S2665913120300583. [cited 2020 Jun 17]

69. Antony J, McGuinness K, Moran K, O'Connor NE. Automatic Detection of Knee Joints and Quantification of Knee Osteoarthritis Severity using Convolutional Neural Networks. Machine Learning and Data Mining in Pattern Recognition MLDM 2017 Lecture Notes in Computer Science [Internet]. 2017;10358. Available from: http://arxiv.org/abs/1703.09856. [cited 2020 Jun 17]

70. Tiulpin A, Klein S, Bierma-Zeinstra SMA, Thevenot J, Rahtu E, van Meurs $J$, et al. Multimodal Machine Learning-based Knee Osteoarthritis Progression Prediction from Plain Radiographs and Clinical Data. Sci Rep. 2019;9:20038.

71. Guan B, Liu F, Haj-Mirzaian A, Demehri S, Samsonov A, Neogi T, et al. Deep learning risk assessment models for predicting progression of radiographic medial joint space loss over a 48-MONTH follow-up period. Osteoarthr Cartil. 2020;28:428-37.
72. Nasser Y, Jennane R, Chetouani A, Lespessailles E, El Hassouni M. Discriminative Regularized Auto-Encoder for Early Detection of Knee OsteoArthritis: Data from the Osteoarthritis Initiative. IEEE Trans Med Imaging. 2020;

73. Neuman P, Dahlberg LE, Englund M, Struglics A. Concentrations of synovial fluid biomarkers and the prediction of knee osteoarthritis 16 years after anterior cruciate ligament injury. Osteoarthritis Cartilage. 2017;25:492-8. Available from: http://www.sciencedirect.com/science/ article/pii/S1063458416302837. [cited 2020 Jun 12]

74. Tiulpin A, Thevenot J, Rahtu E, Lehenkari P, Saarakkala S. Automatic Knee Osteoarthritis Diagnosis from Plain Radiographs: A Deep Learning-Based Approach. Sci Rep. 2018;8:1727.

75. Paixao T, DiFranco MD, Ljuhar R, Ljuhar D, Goetz C, Bertalan Z, et al. A novel quantitative metric for joint space width: data from the Osteoarthritis Initiative (OAI). Osteoarthritis and Cartilage. 2020; Available from: http://www.sciencedirect.com/science/article/pii/S106345842030981X. [cited 2020 Jun 12]

76. Nehrer S, Ljuhar R, Steindl P, Simon R, Maurer D, Ljuhar D, et al. Automated Knee Osteoarthritis Assessment Increases Physicians' Agreement Rate and Accuracy: Data from the Osteoarthritis Initiative. Cartilage. 2019:1947603519888793

77. Oka H, Muraki S, Akune T, Nakamura K, Kawaguchi H, Yoshimura N. Normal and threshold values of radiographic parameters for knee osteoarthritis using a computer-assisted measuring system (KOACAD): the ROAD study. J Orthop Sci. 2010;15:781-9.

\section{Publisher's Note}

Springer Nature remains neutral with regard to jurisdictional claims in published maps and institutional affiliations.
Ready to submit your research? Choose BMC and benefit from:

- fast, convenient online submission

- thorough peer review by experienced researchers in your field

- rapid publication on acceptance

- support for research data, including large and complex data types

- gold Open Access which fosters wider collaboration and increased citations

- maximum visibility for your research: over 100M website views per year

At BMC, research is always in progress.

Learn more biomedcentral.com/submissions 